ARTICLE

Received 3 Nov 2013 | Accepted 10 Mar 2014 | Published 7 Apr 2014

DOI: $10.1038 /$ ncomms4598

\title{
The RNA helicase FRH is an ATP-dependent regulator of CK1a in the circadian clock of Neurospora crassa
}

\author{
Linda Lauinger ${ }^{1}$, Axel Diernfellner ${ }^{1}$, Sebastian Falk ${ }^{2} \&$ Michael Brunner ${ }^{1}$
}

The Neurospora clock protein FRQ forms a complex with casein kinase 1a (CK1a) and FRH, a DEAD box-containing RNA helicase with a clock-independent essential function in RNA metabolism. In the course of a circadian period, FRQ is progressively hyperphosphorylated and eventually degraded. Timed hyperphosphorylation of FRQ is crucial for timekeeping of the clock. Here we show that the ATPase activity of FRH attenuates the kinetics of CK1a-mediated hyperphosphorylation of FRQ. Hyperphosphorylation of FRQ is strictly dependent on site-specific recruitment of a CK1a molecule that is activated upon binding. The FRH ATPase cycle regulates the access of CK1a to phosphorylation sites in FRQ in cis, suggesting that FRH is an ATP-dependent remodelling factor acting on the protein complex. We show that the affinity of CK1a for FRQ decreases with increasing FRQ phosphorylation, suggesting functional inactivation of FRQ in the negative feedback loop of the circadian clock before and independent of its degradation.

\footnotetext{
${ }^{1}$ Biochemistry Centre, University of Heidelberg, Im Neuenheimer Feld 328, Heidelberg D-69120, Germany. ${ }^{2}$ Max Planck Institute of Biochemistry, Am Klopferspitz 18, Martinsried D-82152, Germany. Correspondence and requests for materials should be addressed to M.B.

(email: michael.brunner@bzh.uni-heidelberg.de).
} 
C ircadian clocks are cell-autonomous molecular timing devices that rhythmically organize physiology and behaviour in a daily fashion. An essential aspect of time measurement on the molecular level in eukaryotes is the slowly progressing hyperphosphorylation by casein kinase 1 (CK1) of clock proteins acting in negative feedback loops of the core circadian oscillators ${ }^{1-3}$. Hyperphosphorylation of PERIOD proteins in mammals and flies and FREQUENCY (FRQ) in Neurospora strongly correlates with circadian timekeeping, but molecular mechanisms governing the slow yet precise phosphorylation kinetics of these proteins are poorly understood.

In the course of a day, the Neurospora clock protein FRQ is progressively phosphorylated at $\sim 100$ sites $^{4,5}$. FRQ interacts with $\mathrm{CK} \mathrm{a}^{6}$ and forms a tight complex with FRQ-interacting RNA helicase (FRH), an essential DEAD box-containing RNA helicase ${ }^{7}$. DEAD box-containing RNA helicases comprise a large superfamily of ATPases whose functions are largely unknown ${ }^{8,9}$. Mtr4p, the Saccharomyces cerevisiae orthologue of FRH, is a component of the TRAMP complex and regulates RNA metabolism in cooperation with the nuclear exosome $e^{10-13}$. FRH is essential for the viability of Neurospora owing to its function in RNA metabolism ${ }^{14}$. In addition, FRH has been implicated in a variety of non-essential, clock-related functions: FRH is crucial for cytoplasmic localization and turnover of FRQ ${ }^{15,16}$, and also for the interaction of FRQ with the circadian transcription activator White Collar Complex (WCC) ${ }^{15,17}$. Furthermore, it was reported that FRH mediates turnover of frq RNA by committing the messenger for degradation by the exosome ${ }^{14}$. However, molecular mechanisms underlying these functions are not known. Recently, it has been suggested that the ATPase of FRH is not required for its function in the clock ${ }^{18}$. This conclusion was based on the assumption that dimeric FRQ interacts with only one molecule of FRH, as in the genetic background used the interaction of a FRQ dimer with two molecules of FRH could lead to cross-complementation by two functionally distinct FRH alleles present in the same complex ${ }^{18}$.

Here we analysed the functional interaction of FRQ with FRH and CK1a in Neurospora, in a heterologous system in vivo and in a cell-free system with recombinant components. Our data demonstrate that the native FRQ-FRH complex (FFC) contains two FRH and two FRQ molecules. We show that the ATPase of FRH regulates the kinetics of hyperphosphorylation of FRQ by bound CK1a.

\section{Results}

Characterization of the FFC. The polypeptide chains of FRQ and FRH have predicted molecular masses $\left(M_{\mathrm{r}}\right)$ of 108 and $125 \mathrm{kDa}$, respectively. We analysed the interaction of FRQ with FRH and the stoichiometry of the components by gel filtration. The native FFC had an apparent $M_{\mathrm{r}}$ of $\sim 670 \mathrm{kDa}$. Although all FRQ is bound to $\mathrm{FRH}^{7}$, only $\sim 15 \%$ of FRH were in complex with FRQ (Fig. 1a and Supplementary Fig. 1a). The fraction of FRH that is not associated with FRQ may serve non-circadian functions that are essential for the viability of Neurospora ${ }^{7}$. In constant darkness, when FRQ was rhythmically expressed, FRH levels remained constant (Supplementary Fig. 1b), indicating that the circadian clock does not control its expression level.

FRQ forms a dimer or oligomer via its N-terminal coiled-coil domain $^{19}$. We found that deletion of the nuclear localization signal (NLS) of $\mathrm{FRQ}^{20}$ that is located next to the coiled-coil domain (Fig. 1b) did not affect its interaction with FRH but compromised oligomerization of FRQ $\triangle$ NLS (deletion of residues 194-199): Thus, FRH co-immunoprecipitated with FRQ $\Delta$ NLS (Fig. 1c) and the complex had a $M_{\mathrm{r}}$ of $\sim 250 \mathrm{kDa}$ (Fig. $1 \mathrm{~d}$ and Supplementary Fig. 1c), consistent with an assembly of one molecule FRQ $\Delta$ NLS with one molecule FRH. Accordingly, the wild-type (WT) complex should also contain equimolar amounts of FRQ and FRH. As FRQ is a dimer (or oligomer), the FFC $(\sim 670 \mathrm{kDa})$ should contain at least two molecules of FRQ and two molecules of FRH.

To further address the stoichiometry of the FFC, we expressed FRQ, FRH and an N-terminally truncated FRH $(\mathrm{FRH} \Delta \mathrm{N})$ in Escherichia coli and purified the recombinant proteins (Supplementary Fig. 1d). FRQ formed a tight complex with FRH $\triangle N$ (used for better separation from FRQ by SDS-PAGE), demonstrating that complex formation does not require ATP binding or hydrolysis by FRH $\Delta \mathrm{N}$ (Fig. 1e). The complex between FRQ and FRH $\Delta \mathrm{N}$ eluted from a gel filtration with an apparent $M_{\mathrm{r}}$ $>670 \mathrm{kDa}$ was similar to the native Neurospora FFC. The complex between FRQ and FRH $\Delta \mathrm{N}$ (Fig. 1e) as well as the complex of FRQ with a purified glutathion-S-transferase (GST)tagged FRH (Supplementary Fig. 1e) contained apparently stoichiometric amounts of both proteins, supporting that the native complex is composed of a dimer or even trimer of FRQFRH protomers.

It has been suggested that the FFC facilitates rhythmic turnover of $f r q \mathrm{RNA}^{14}$. The mutant $f r q^{9}$ allele expresses full-length RNA but, due to a frameshift mutation, it encodes a truncated $\mathrm{FRQ}^{9}$ protein that does not associate with $\mathrm{FRH}^{14}$. We inhibited transcription in $W T$ and $f r q^{9}$ strains with thiolutin ${ }^{14}$ and found that the turnover kinetics of $f r q$ and $f r q^{9}$ RNA was similar (Supplementary Fig. 2a), suggesting that the FFC may not regulate turnover of $f r q$ RNA.

To assess the function of the ATPase of FRH in the circadian clock, we mutated the Walker A and DEXH box motifs (Supplementary Fig. 2b), elements required for ATP binding and hydrolysis, respectively ${ }^{21,22}$. As FRH is essential for the viability of Neurospora ${ }^{7}$, we expressed mutant and WT versions of FLAG-tagged FRH in an $\mathrm{frh}^{+}$background. The FLAG-tagged FRH versions comprised $\sim 20 \%$ of the total FRH (Fig. $2 a$ ) and did neither affect FRQ expression levels nor circadian rhythms (Supplementary Fig. 2c). FLAG-immunoprecipitation (FLAG-IP) revealed that substantially less FRQ was in complex with the

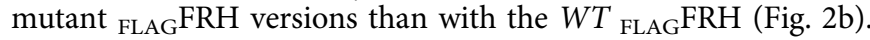
When protein synthesis was inhibited with cycloheximide, FRQ in complex with the ${ }_{\text {FLAGFRH (followed by FLAG-IP) was }}$ degraded with a half-time of $3.5 \mathrm{~h}$ (Fig. 2c), which corresponds to the previously reported degradation kinetics of FRQ ${ }^{6,23}$. FRQ in complex with Walker A and DEAD box mutants of ${ }_{\text {FLAG }} \mathrm{FRH}$ was degraded significantly faster (Fig. 2c). Thus, the turnover of FRQ is directly or indirectly modulated by ATP binding and hydrolysis of FRH.

Expression and analysis of Neurospora clock proteins in yeast. We expressed FRQ, FRH and CK1a in S. cerevisiae to study their interaction and the phosphorylation of FRQ independent of the essential functions of CK1a and FRH in Neurospora. When FRQ was expressed in the absence of FRH and CK1a, it accumulated at a low level and in hypophosphorylated form (Fig. 3a). Thus, yeast kinases do not support hyperphosphorylation of FRQ (we define hypo- and hyperphosphorylation operationally by small and large shifts, respectively, in the electrophoretic mobility of FRQ). Coexpression with FRH resulted in elevated expression levels of FRQ, and its phosphorylation state was slightly lower than in the absence of FRH (Fig. 3a and Supplementary Fig. 3a). This demonstrates that FRH stabilizes FRQ and suppresses low-level phosphorylation of FRQ by yeast kinases. Mtr4p, the yeast orthologue of $\mathrm{FRH}^{7}$, does not interact with FRQ (Supplementary Fig. 3b) and does not substitute for the stabilizing function of FRH. When co-expressed with CK1a, FRQ accumulated at a low 


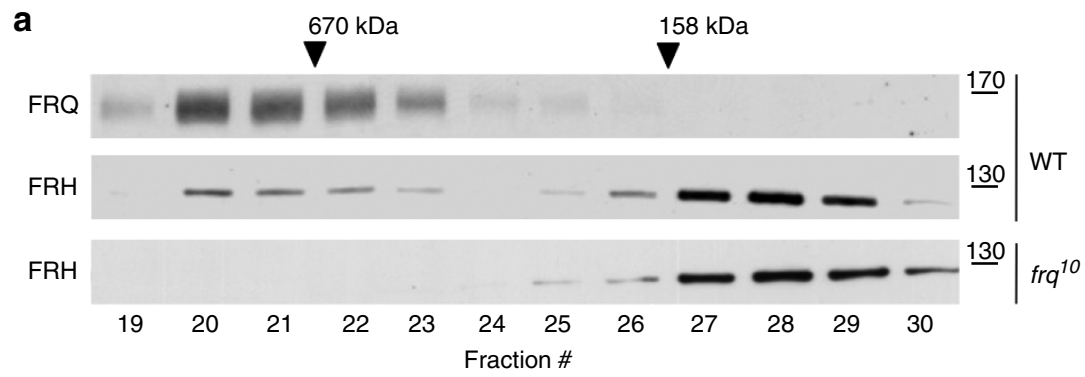

b

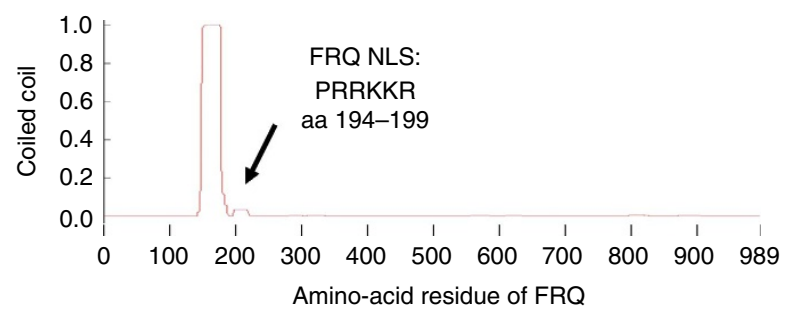

C
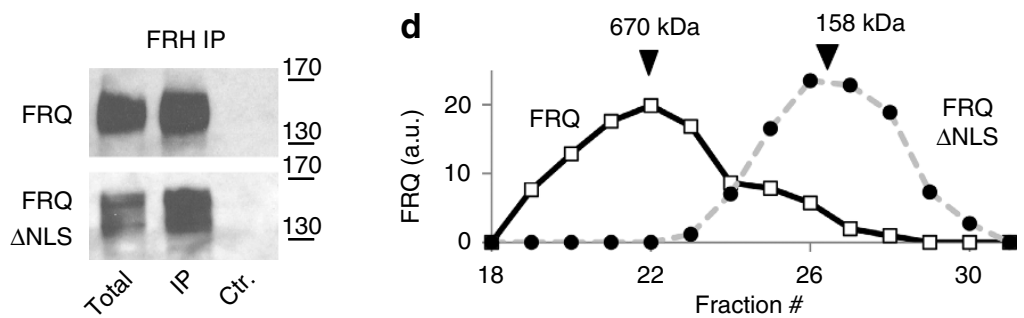

e

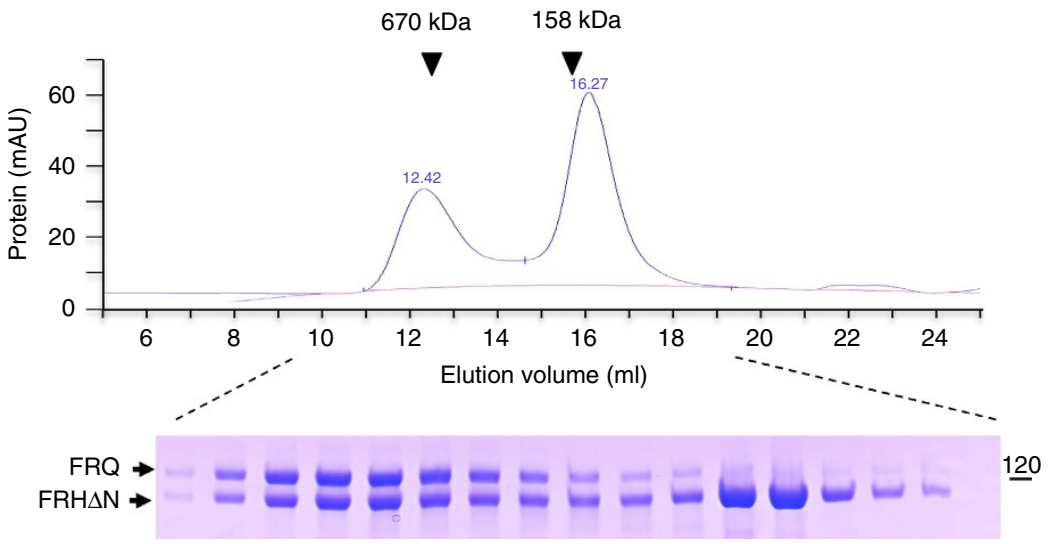

Figure 1 | Composition of the FFC. (a) Gel filtration analysis (Superose 6) of FRQ (108 kDa) and FRH (125 kDa) from wild-type (WT) and fra ${ }^{10}$ strains. The elution of molecular mass standards is indicated. (b) Prediction of coiled-coils (Expasy) in FRQ (989 amino acid residues). Dimerization/ oligomerization of FRQ is dependent on the coiled-coil domain ${ }^{19}$. The location and composition of the NLS are indicated. (c) FRQ $\Delta N L S$ forms a stable complex with FRH. FRH from WT and frq $\Delta$ NLS strains was immunoprecipitated with affinity-purified antibodies. Western blots were decorated with FRQ antibody to detect co-immunoprecipitation. (d) FRQ $\Delta \mathrm{NLS}^{20}$ does not form oligomeric complexes. Gel filtration (Superose 6) analysis of native protein extracts of light-grown WT and frq $\triangle N L S$ strains. Densitometric quantification of western blots (Supplementary Fig. 1c) showing the elution profiles of FRQ and FRQ $\Delta$ NLS. (e) In vitro reconstitution of the FFC. Recombinant FRQ and FRH $\Delta N$ (Supplementary Fig. 1d) form a $\sim 670-k D a$ assembly with approximately 1:1 stoichiometry. Gel filtration (Superose 6) and SDS-PAGE analysis of the elution profiles of FRQ and FRH $\Delta \mathrm{N}$ are shown.

level and in a hyperphosphorylated form (Fig. 3a). Finally, when co-expressed with both, CK1a and FRH, steady-state levels of FRQ were elevated and it was heterogeneously phosphorylated, reminiscent to the steady state of FRQ in light-grown Neurospora (Fig. 3a and Supplementary Fig. 3c). The data show that FRH attenuates CK1a-dependent hyperphosphorylation of FRQ.

FRQ is an intrinsically unfolded protein ${ }^{23}$. We asked whether FRH and CK1a affect the turnover of FRQ in yeast. The degradation kinetics of FRQ in the absence of FRH was fast $\left(t_{1 / 2}\right.$ $\sim 0.5 \mathrm{~h}$ ), regardless of whether CK1a was present or absent
(Fig. 3b). Thus, CK1a-dependent hyperphosphorylation of FRQ is not rate determining for degradation of FRQ in the absence of FRH. In the presence of FRH but absence of CK1a, FRQ was rather stable $\left(t_{1 / 2} \sim 4 \mathrm{~h}\right)$ (Fig. $3 \mathrm{~b}$ ), confirming that FRH stabilizes $\mathrm{FRQ}^{18}$. Finally, in the presence of FRH and CK1a, FRQ displayed intermediate stability $\left(t_{1 / 2} \sim 1.5 \mathrm{~h}\right)$ (Fig. $\left.3 \mathrm{~b}\right)$. Together, the data suggest that the turnover of FRQ in complex with FRH is regulated by CK1a-dependent hyperphosphorylation.

The FRQ6B2 mutant, in which amino-acid residues 774-776 were exchanged by alanyl residues, is compromised in complex 


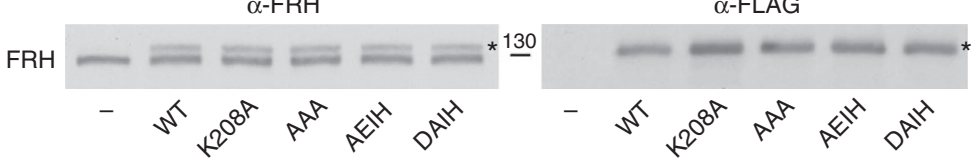

b

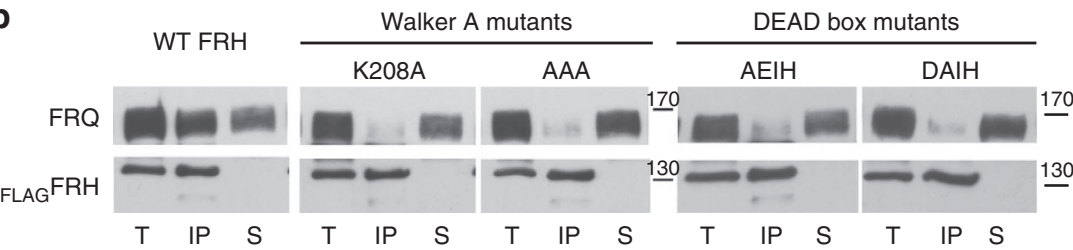

C
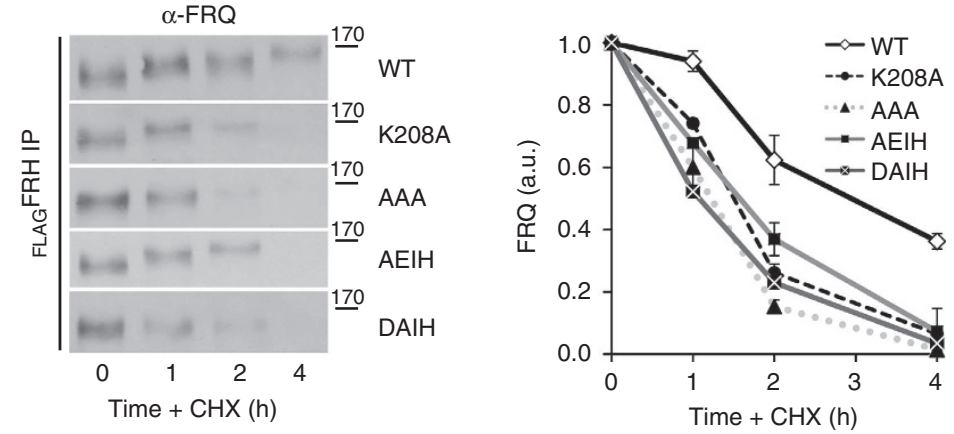

Figure 2 | Characterization of the FRQ-FRH interaction. (a) Western blot analysis of the indicated FLAG-tagged FRH versions expressed in a frh ${ }^{+}$ background. WT: FLAGFRH; K208A and AAA: Walker A box mutants; AEIH and DAIH: DEAD box mutants. FRH antibody and FLAG antibody was used for decoration in the left and right panel, respectively. Asterisks indicate FLAG-tagged FRH. (b) FRQ does not stably accumulate in complex with ATPase and DEAD box mutants of FRH. Co-IP of FRQ with ${ }_{F L A G} F R H$ versions pulled down with M2 FLAG Sepharose is shown. Protein extracts of light-grown cultures expressing WT and mutant versions of FLAG-tagged FRH were used. Western blots of the total protein extract (T), the immunoprecipitate (IP) and the supernatant (S) were decorated with FRQ antibody (upper panels) and FLAG antibody (lower panels). (c) Turnover kinetics of FRQ in complex with FLAG-FRH versions. Light-grown cultures were incubated with cycloheximide (CHX). Samples were harvested at the indicated time points and the indicated ${ }_{F L A G} F R H$ versions were immunoprecipitated (FLAG-IP). Immunoprecipitates of mutant FRH versions are five-fold overrepresented. Co-IP of FRQ was detected by western blotting (left panel) and quantified by densitometry (right panel). FRQ levels at time 0 were set equal to 1 (a.u., arbitrary units). Error bars indicate s.d. $(n=3)$.

formation with $\mathrm{FRH}^{15}$. When expressed in $S$. cerevisiae, FRQ6B2 was hypophosphorylated in the absence of CK1a and hyperphosphorylated in the presence of CK1a (Fig. 3c). Coexpression of FRH did neither support accumulation of elevated levels of FRQ6B2 nor attenuate its CK1a-dependent hyperphosphorylation. Thus, association with FRH is required for the stabilization of FRQ and for the modulation of its CK1adependent phosphorylation state.

The interaction of FRQ with CK1a is dependent on FCD1 and FCD2, two short putative coiled-coil motifs that interact with each other over a long distance to form directly or indirectly a binding site for CK1a ${ }^{23}$. When FRQ $\triangle F C D 1$ and FRQ $\triangle F C D 2$, deletion mutants lacking one of these motifs, were co-expressed with CKla, the mutant FRQ versions remained hypophosphorylated (Fig. 3d). Thus, despite the expression of high levels of CK1a in yeast, binding of CK1a to FRQ is required for efficient hyperphosphorylation. We then co-expressed FRQ with predicted catalytically inactive versions of CK1a. The mutant kinases CK1aK41R and CK1aD131N ${ }^{24,25}$ associated with FRQ (Supplementary Fig. 3d) but did not support hyperphosphorylation of FRQ (Fig. 3e), demonstrating that CK1a does not simply fulfil a structural role in the complex.

We then co-expressed FRQ with Walker A and DEXH box mutants of FRH, respectively. The mutant and WT versions of FRH associated with FRQ (Supplementary Fig. 3e). The mutant versions of FRH stabilized FRQ in the absence and presence of CK1a but did not attenuate hyperphosphorylation of FRQ in the presence of CK1a (Fig. 3f). Expression levels of CK1a were similar in all strains, indicating that the attenuated phosphorylation of FRQ in the presence of WT FRH was not limited by CK1a (Supplementary Fig. 3f). Thus, the association with catalytically inactive FRH is sufficient for the protection of FRQ against degradation, while ATP binding and hydrolysis by FRH regulates CK1a-dependent hyperphosphorylation of FRQ.

Phosphorylation of FRQ in cell-free extracts. We next characterized phosphorylation of FRQ by recombinant CK1a in a cellfree system ${ }^{26}$. Light-grown Neurospora expresses high levels of heterogeneously phosphorylated FRQ. When a native protein extract (cytosol) of such mycelia was incubated with recombinant His $6 \mathrm{CK} 1 \mathrm{a}$ at $30^{\circ} \mathrm{C}$, the endogenous FRQ was specifically hyperphosphorylated in a concentration-dependent manner (Fig. 4a). Efficient hyperphosphorylation of FRQ was observed at rather high ${ }_{\mathrm{His} 6} \mathrm{CK} 1 \mathrm{a}$ concentration when the recombinant kinase was present in huge molar excess over FRQ. Notably, endogenous CK1a present in the Neurospora cytosol did not support hyperphosphorylation at $30^{\circ} \mathrm{C}$, although FRQ can be efficiently immunoprecipitated in a complex with endogenous $\mathrm{CK} 1 \mathrm{a}$ at $4{ }^{\circ} \mathrm{C}$ (refs 6,27). To characterize the temperaturedependent-binding equilibrium of CK1a and FRQ, we analysed their interaction by immunoprecipitation at 4 and $30^{\circ} \mathrm{C}$ (Fig. 4b). FRQ co-immunoprecipitated efficiently with CK1a at $4{ }^{\circ} \mathrm{C}$, whereas the interaction was less efficient at $30^{\circ} \mathrm{C}$, indicating that the dissociation of the CK1a-FRQ interaction is rather fast at physiological temperatures. FLAG-tagged CK1a displayed similar 


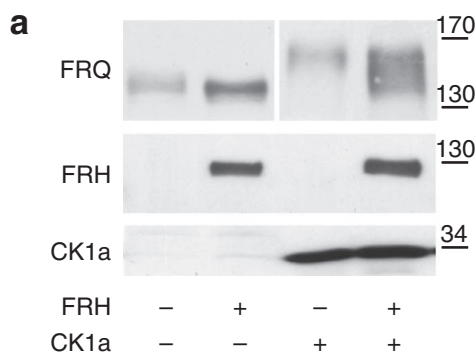

b
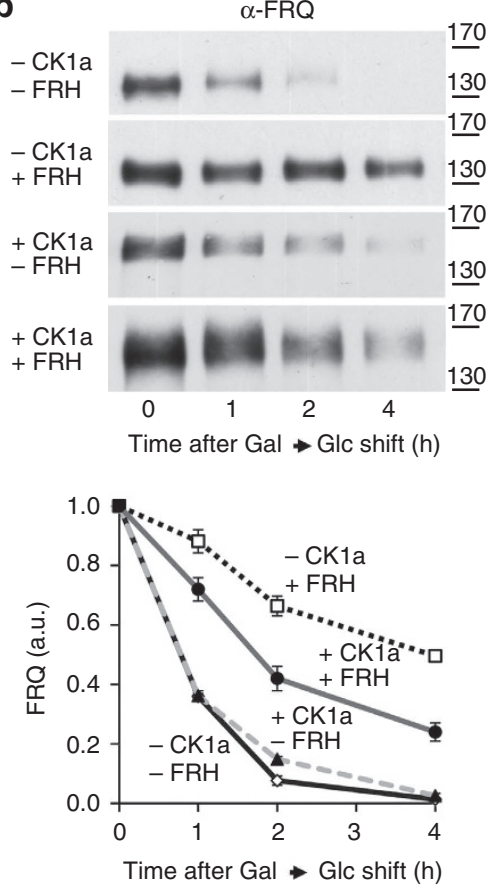

C

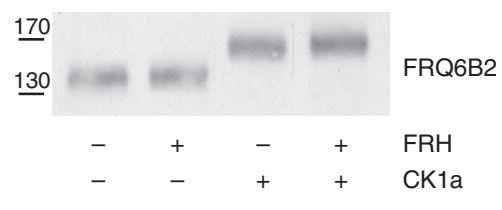

d

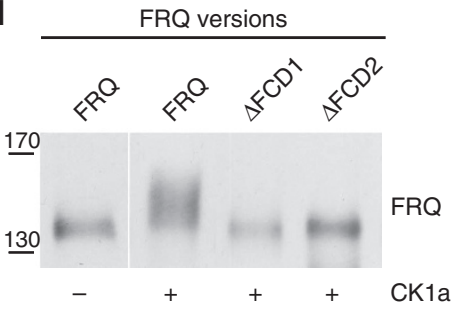

e

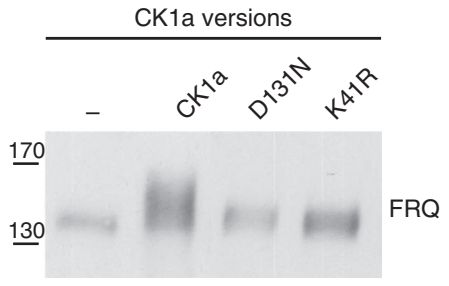

f

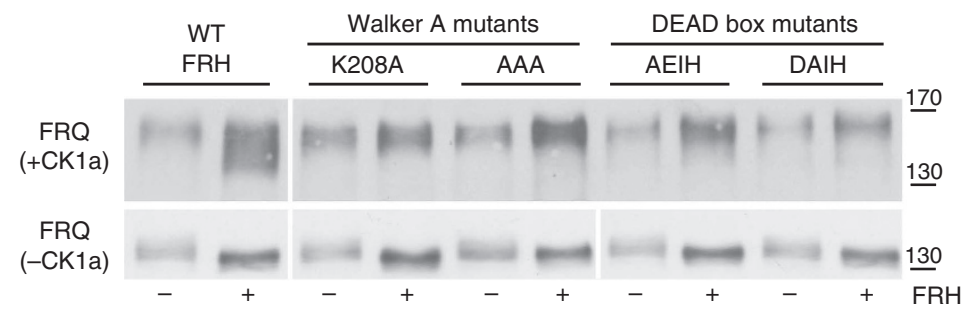

Figure 3 | Expression and analysis of Neurospora clock proteins in yeast. (a) FRH stabilizes FRQ and attenuates its CK1a-dependent hyperphosphorylation. FRQ, FRH and CK1a were expressed in S. cerevisiae and expression levels and phosphorylation state were analysed by western blot. (b) The degradation kinetics of FRQ is dependent on FRH and CK1a. S. cerevisiae strains expressing FRQ under control of the GAL1 promoter together with $\mathrm{CK} 1 \mathrm{a}$ and $\mathrm{FRH}$ when indicated were shifted from galactose to glucose containing medium. Samples were harvested at the indicated time points and analysed by immunoblotting with FRQ antibodies (upper panel). FRQ levels were quantified by densitometry (lower panel). Error bars indicate s.d. ( $n=3$ ). It should be noted that the apparent degradation kinetics includes dilution of FRQ because of growth of the yeast cells. (c) Expression levels in yeast and phosphorylation state of FRQ6B2 (ref. 14) are independent of FRH. FRQ6B2 is a mutant that does not interact with FRH. (d) CK1a-dependent phosphorylation of FRQ is dependent on the CK1a interaction domains FCD1 and FCD2 of FRQ. Western blot analysis of the phosphorylation state of indicated FRQ versions expressed in yeast. Co-expression of CK1a is indicated. (e) The phosphorylation state of FRQ is dependent on expression of catalytically active CK1a. FRQ was co-expressed in yeast with active CK1a and with the catalytically inactive versions D131N and K41R as indicated. (f) ATP binding and hydrolysis by FRH regulates $\mathrm{CK} 1 \mathrm{a}$-dependent hyperphosphorylation of $\mathrm{FRQ}$ but the ATPase activity of FRH is not required for the stabilization of FRQ. FRQ was expressed without and with FRH versions as indicated. Upper panel: CK1a was co-expressed. Lower panel: No co-expression of CK1a. FRQ expression level and phosphorylation state were analysed by western blotting. Hyper- and hypophosphorylated FRQ accumulated in the presence (upper panel) and absence (lower panel) of CK1a, respectively. Hyperphosphorylation was attenuated by WT FRH but not by the FRH mutants.

temperature-dependent binding to FRQ (Supplementary Fig. 4a). To analyse the temperature dependence of the activity of CKla, we used $\beta$-casein as a substrate. As expected, the catalytic activity of recombinant ${ }_{\text {His } 6} \mathrm{CK} 1 \mathrm{a}$ was higher at $30^{\circ} \mathrm{C}$ than at $4{ }^{\circ} \mathrm{C}$ (Fig. 4c). We then prepared Neurospora cytosol from light-grown cultures, containing heterogeneously phosphorylated FRQ or cytosol from light-induced cultures containing hypophosphorylated FRQ. The cytosols were 
a

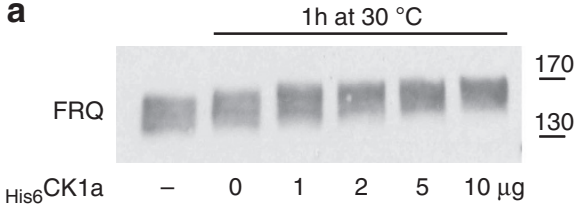

b
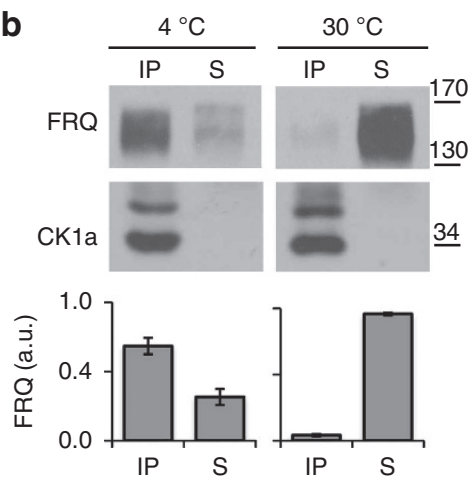

d

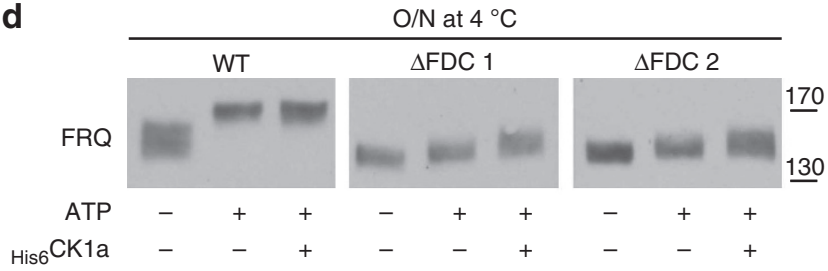

e

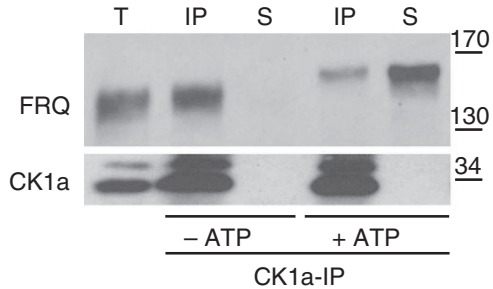

Figure 4 | Phosphorylation of FRQ in cell-free extracts. (a) CK1a-

dependent hyperphosphorylation of FRQ in a cell-free system. Native protein extract of light-grown Neurospora expressing heterogeneously phosphorylated FRQ (lane 1) was incubated for $1 \mathrm{~h}$ at $30^{\circ} \mathrm{C}$ with the indicated amounts of recombinant ${ }_{\text {His } 6} \mathrm{CK}$ la. The phosphorylation state of FRQ was dependent on the His6 $\mathrm{CK} 1 \mathrm{a}$ concentration. (b) Temperature-dependent interaction of CK1a and FRQ. Native cytosol was prepared from WT and CK1a was immunoprecipitated at 4 and $30^{\circ} \mathrm{C}$. Aliquots of IP and supernatant (S) were analysed by western blot. FRQ and CKla signals were quantified by densitometry. Error bars indicate s.d. $(n=3)$. FRQ co-immunoprecipitated with higher efficiency at $4{ }^{\circ} \mathrm{C}$ than at $30^{\circ} \mathrm{C}$. (c) Temperature dependence of ${ }_{\mathrm{His} 6} \mathrm{CKla}$ activity. Recombinant

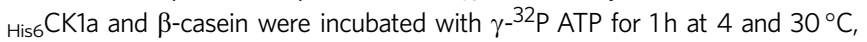
respectively. Phosphorylation of $\beta$-casein and autophosphorylation of CK1a was detected by autoradiography and quantified. Error bars indicate s.d. $(n=3)$ (d) Hyperphosphorylation of FRQ in vitro is dependent on CKla interaction domains (FCDs) even at high ${ }_{\mathrm{His} 6} \mathrm{CK} 1 \mathrm{a}$ concentration. Protein extracts from cultures expressing WT FRQ and FRQ $\triangle F C D 1$ and FRQ $\triangle F C D 2$, respectively, were incubated overnight at $4{ }^{\circ} \mathrm{C}$ in the absence or presence of ATP and purified recombinant ${ }_{\mathrm{His}} \mathrm{CK}$ Ca as indicated. The phosphorylation state of FRQ was analysed by immunoblotting. (e) Hyperphosphorylation of FRQ reduces its affinity for CK1a. CK1a was immunoprecipitated overnight at $4{ }^{\circ} \mathrm{C}$ from WT cytosol (containing endogenous FRQ and CKla) in the absence or presence of ATP. Without ATP, FRQ remained heterogeneously phosphorylated and co-immunoprecipitated efficiently with CK1a. With ATP, FRQ was hyperphosphorylated and its association with CKla was reduced. Total protein extract before incubation $(T)$, the IP and the supernatant $(S)$ are shown. A three-fold excess of IP and $\mathrm{S}$ were loaded with respect to $\mathrm{T}$. incubated overnight in the presence of ATP without and with additional recombinant ${ }_{\mathrm{His} 6} \mathrm{CK} 1 \mathrm{a}$ at $4{ }^{\circ} \mathrm{C}$ to stabilize the interaction of CK1a with FRQ. Under these conditions, heterogeneously as well as hypophosphorylated FRQ was efficiently hyperphosphorylated in the presence and also in the absence of additional recombinant ${ }_{\text {His6 }} \mathrm{CK} 1 \mathrm{a}$ (Fig. $4 \mathrm{~d}$, left panel and Supplementary Fig. $4 \mathrm{~b}$ ), indicating that the concentration of endogenous CK1a was sufficient for hyperphosphorylation of FRQ under conditions that favour binding of the kinase to FRQ. We then prepared cytosol from strains expressing FRQ versions lacking the CK1a interaction domains FCD1 and FCD2. When these cytosols were incubated with ATP at $4{ }^{\circ} \mathrm{C}$, the FRQ $\Delta \mathrm{FCD}$ versions were neither hyperphosphorylated by endogenous CK1a nor by high amounts of recombinant ${ }_{\mathrm{His} 6} \mathrm{CK} 1 \mathrm{a}$ (Fig. $4 \mathrm{~d}$ ). The data demonstrate that FRQ is hyperphosphorylated in cis by bound $\mathrm{CK} 1 \mathrm{a}$. The reaction is limited by the binding equilibrium of CK1a and FRQ (strengthened at $4^{\circ} \mathrm{C}$ ) rather than by the catalytic activity of $\mathrm{CK} 1 \mathrm{a}$ (higher at $30^{\circ} \mathrm{C}$ ). Free ${ }_{\text {His }} \mathrm{CK} 1 \mathrm{a}$, even in huge molar excess and in high concentration, does not support efficient phosphorylation, indicating that FRQ is a rather poor substrate for free CK1a.

We then asked whether the phosphorylation state of FRQ affects its interaction with CK1a. Neurospora cytosol was incubated overnight without and with ATP, and CKla was then immunodepleted (Fig. 4e). In the absence of ATP, FRQ remained heterogeneously phosphorylated and co-immunoprecipitated efficiently with CK1a. In the presence of ATP, FRQ was hyperphosphorylated and its co-immunoprecipitation with CK1a was severely reduced. The data indicate that binding of CKla to FRQ is compromised by hyperphosphorylation of FRQ. As FRQ recruits CK1a to phosphorylate and inactivate its transcription activator WCC, the data suggest that the hyperphosphorylation-dependent loss of CK1a functionally inactivates the FFC as a circadian inhibitor, which should relieve the negative feedback in the core oscillator independent of and before the degradation of FRQ.

Reconstitution of Neurospora clock protein interactions in vitro. To characterize the phosphorylation of FRQ in vitro, we incubated recombinant FRQ with ${ }_{\mathrm{His} 6} \mathrm{CK} 1 \mathrm{a}$ and ATP at $30^{\circ} \mathrm{C}$. Under these conditions, FRQ was phosphorylated only to a limited extent and not hyperphosphorylated (Fig. 5a). Members of the CK1 protein family catalyse de novo phosphorylation of serine and threonine residues in a negatively charged environment and, in addition, phosphate-directed phosphorylation of $\mathrm{S} / \mathrm{T}_{\mathrm{P}} \mathrm{X}_{2 / 3} \mathrm{~S} / \mathrm{T}$ motifs that were previously phosphorylated by a priming kinase $\mathrm{e}^{28}$. To allow priming phosphorylation, we incubated recombinant $\mathrm{FRQ}$ at $30^{\circ} \mathrm{C}$ with and without purified ${ }_{\mathrm{His}} \mathrm{CK} 1 \mathrm{a}$ in the presence of either cytosol of a $\Delta \mathrm{frq}$ strain or yeast cytosol (Fig. 5b). In the presence of cytosol but in the absence of His6 CK1a, recombinant FRQ was hypophosphorylated, demonstrating that neither Neurospora kinases (including endogenous CK1a) nor yeast kinases supported efficient hyperphosphorylation of recombinant FRQ under these conditions. However, when ${ }_{\text {His6 }} \mathrm{CK} 1 \mathrm{a}$ was added together with Neurospora or yeast cytosol, FRQ was efficiently hyperphosphorylated (Fig. 5b), suggesting that the cytosols supported in promiscuous manner priming phosphorylation of FRQ, which then served as substrate for His6 CK1a. Hyperphosphorylation of FRQ was dependent on the concentration of ${ }_{\mathrm{His}} \mathrm{CK} 1 \mathrm{a}$, despite molar excess of the kinase over recombinant FRQ (Supplementary Fig. 5a), suggesting a limitation of the reaction by the binding equilibrium of ${ }_{\text {His } 6} \mathrm{CK} 1 \mathrm{a}$ and FRQ. Recombinant FRQ was then incubated with ATP and $\Delta f r q$ cytosol at 4 and $30^{\circ} \mathrm{C}$ to strengthen and weaken, respectively, complex formation of FRQ with the 
a

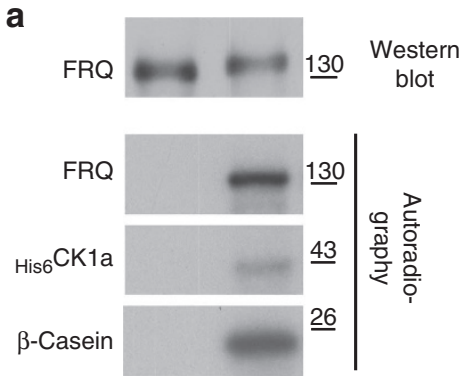

b

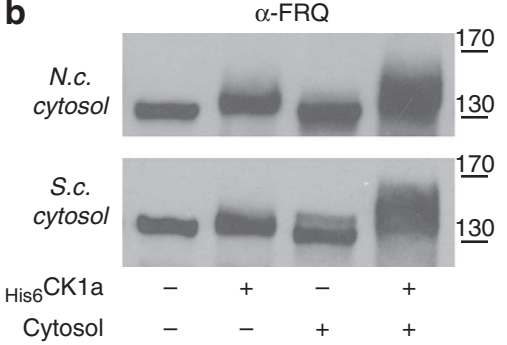

C

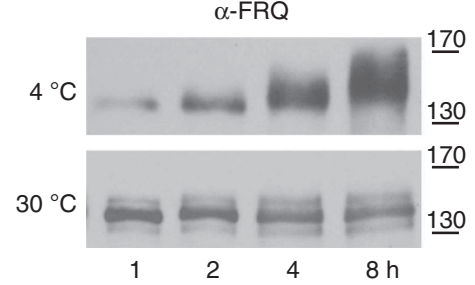

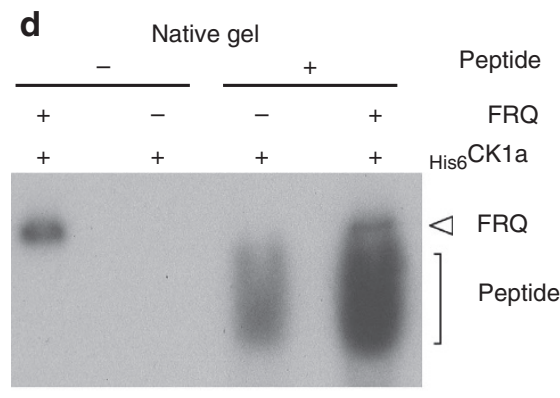

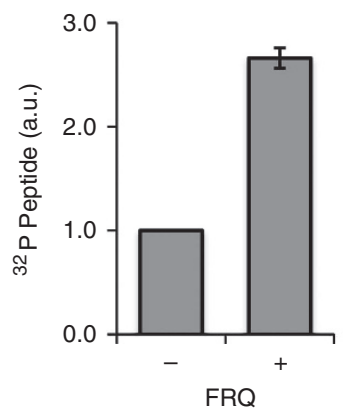

Figure 5 | Functional interaction of recombinant FRQ and CK1a in vitro. (a) His6 CK1a phosphorylates recombinant FRQ only to a limited extend. Purified recombinant FRQ $(100 \mathrm{ng})$ and ${ }_{\mathrm{His} 6} \mathrm{CK} 1 \mathrm{a}(5 \mu \mathrm{g})$ were incubated with $\gamma^{3}{ }^{32} \mathrm{P}$ ATP for $1 \mathrm{~h}$ at $30^{\circ} \mathrm{C}$. The upper panel shows a western blot analysis of FRQ. The lower panels are autoradiograms showing incorporation of ${ }^{32} \mathrm{P}$ into recombinant $\mathrm{FRQ}$, His6 $\mathrm{CK}$ la (autophosphorylation) and $\beta$-casein (CK1a activity control). (b) Hyperphosphorylation of recombinant FRQ by ${ }_{\text {His } 6} \mathrm{CK} 1 \mathrm{a}$ is dependent on priming kinases supplied by cytosol of Neurospora crassa

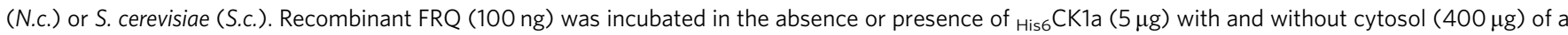
N.c. $\mathrm{frq}^{10}$ ( $\Delta \mathrm{frq}$ ) strain or yeast cytosol when indicated. The samples were incubated $1 \mathrm{~h}$ at $30^{\circ} \mathrm{C}$. (c) CK1a present in Neurospora cytosol supports hyperphosphorylation of recombinant FRQ at $4{ }^{\circ} \mathrm{C}$ but not at $30^{\circ} \mathrm{C}$. Purified FRQ $(100 \mathrm{mg})$ was incubated with fra $q^{10}$ cytosol for the indicated time periods. (d) CK1a is activated by recruitment to FRQ. His 6 CK1a (100 ng) was pre-incubated for 30 min at $4{ }^{\circ} \mathrm{C}$ with a four-fold molar excess of recombinant FRQ $(1 \mu \mathrm{g})$ to allow stable complex formation or with $1 \mu \mathrm{g}$ BSA to balance the total protein content. Subsequently, the phosphopeptide Ac-KRRRALS(pS)VASLdP(Sox)G-NH $\left(2 \mu\right.$ g) (Life Technologies) was incubated at $4{ }^{\circ} \mathrm{C}$ with $\gamma^{-32 P}$ ATP $(25 \mu \mathrm{M})$ and with either the preformed ${ }_{\text {His } 6} \mathrm{CK} 1 \mathrm{a}-\mathrm{FRQ}$ complex or the corresponding amount of free ${ }_{\mathrm{His} 6} \mathrm{CK} 1 \mathrm{a}(+\mathrm{BSA})$. Reactions were terminated after the indicated time periods and analysed by native-PAGE and autoradiography (upper panel). FRQ and the peptide were phosphorylated. BSA was not phosphorylated and autophosphorylation of CK1a is not visible at the shown exposure. Lower panel: phosphorylation of the peptide was quantified by densitometry. Error bars indicate s.d. $(n=3$ ).

endogenous CK1a present in the Neurospora cytosol (Fig. 5c). FRQ was hyperphosphorylated at $4{ }^{\circ} \mathrm{C}$ but not at $30^{\circ} \mathrm{C}$, supporting that the reaction was limited by the binding equilibrium of endogenous CK1a to recombinant FRQ.

We then asked whether CK1a is activated by recruitment to FRQ. To efficiently drive complex formation, CK1a was incubated at $4{ }^{\circ} \mathrm{C}$ with an $\sim$ four-fold molar excess of recombinant FRQ. Subsequently, we incubated a CK1 phosphopeptide substrate (Fig. 5d) or $\beta$-casein (Supplementary Fig. 5b) at $4^{\circ} \mathrm{C}$ with either the preformed ${ }_{\mathrm{His} 6} \mathrm{CK} 1 \mathrm{a}-\mathrm{FRQ}$ complex or with the corresponding amount of free ${ }_{\mathrm{His} 6} \mathrm{CK} 1 \mathrm{a}$. Both substrates were more efficiently phosphorylated by the preformed ${ }_{\mathrm{His} 6} \mathrm{CK} 1 \mathrm{a}-\mathrm{FRQ}$ complex than by free ${ }_{\mathrm{His} 6} \mathrm{CK} 1 \mathrm{a}$ (Fig. $5 \mathrm{~d}$ and Supplementary Fig. 5b). The data suggest that the catalytic site of FRQ-associated CK1a is accessible for substrate in cis and trans. Moreover, CK1a is activated by recruitment to FRQ. In this way, a single molecule of bound CK1a could phosphorylate in cis a large number of sites that are distributed all over the intrinsically disordered polypeptide chain of FRQ and also WCC that is recruited to the FFC $^{16,17,29}$

\section{Discussion}

Progressive hyperphosphorylation is a hallmark of clock proteins in all eukaryotic model systems and correlates strongly with circadian timing. We show here that FRH, the Neurospora orthologue of the S. cerevisiae RNA helicase MTR4, modulates the slowly progressing phosphorylation of FRQ on a circadian timescale. FRH associates with and stabilizes FRQ in ATPindependent manner, while the ATPase of FRH attenuates the kinetics of CK1a-dependent hyperphosphorylation of FRQ. The regulatory function of FRH in the FRQ complex is independent of its essential RNA helicase activity in the TRAMP complex and exosome pathway, and hence, FRH has two mechanistically distinct functions.

In vitro studies with recombinant FRQ and FRH as well as analysis of the Neurospora clock proteins in S. cerevisiae indicate that the FRQ-FRH interaction is tight and is not dependent on ATP, but it is not known whether the ATPase of FRH modulates a transient dissociation-association cycle.

We show that hyperphosphorylation of FRQ is dependent on priming kinases and on CK1a. Priming phosphorylation of FRQ 
is provided by $S$. cerevisiae, suggesting that the priming kinases are rather promiscuous. In Neurospora, FRQ is phosphorylated by CKII (ref. 30), calcium/calmodulin-dependent kinase ${ }^{31}$ and protein kinase $\mathrm{A}^{32}$. Hence these kinases may, in addition to their putative specific roles, serve as priming kinases. In contrast to priming phosphorylation, subsequent hyperphosphorylation of FRQ is strictly dependent on Neurospora CK1a. The phosphorylation reaction is limited by the FRQ-CK1a-binding equilibrium. Free CK1a is rather inactive and becomes catalytically activated by recruitment to FRQ via FCD1 and FCD2. The FRQ-dependent activation of CK1a did not require FRH. Interestingly, it has recently been shown that CK1E, a mammalian homologue of CK1a, is activated by recruitment to the human RNA helicase DDX3 to phosphorylate its substrate dishevelled in a wnt/ $\beta$-catenin signalling complex ${ }^{33}$. Like the FFC, the wnt $\beta$-catenin signalling complex contains a DEAD box helicase, $C K 1$ and substrate. CK1 $1 \varepsilon$ is recruited to the complex by DDX3 (the helicase) while CK1a is recruited by FRQ (the substrate). In both systems, CK1, a simple monomeric enzyme, is rather inactive and is activated by site-specific recruitment to a target complex. The co-occurrence of a DEAD box helicase and CK1 in both, the Neurospora clock complex and in the wnt $\beta$-catenin signalling complex ${ }^{33}$ may suggest a conserved functional interaction of RNA helicases and CK1 in protein complexes. However, while the ATPase of FRH regulates the activity of CK1a, a putative regulatory function of the conserved ATPase of DDX in the wnt/ $\beta$-catenin pathway has not been uncovered.

How can one CK1a molecule that is bound to a specific site in FRQ facilitate phosphorylation of a large number of sites distributed throughout the polypeptide chain ${ }^{4,5}$ ? FRQ is an intrinsically unfolded protein ${ }^{18,23}$. The few predicted folded segments correspond to the $\mathrm{N}$-terminal coiled-coil domain that facilitates oligomerization of FRQ, the two FCDs in the central portion, which interact with each other via a coiled-coil and the FRH interaction domain in the C-terminal portion of $\mathrm{FRQ}^{7,19,23}$. The remaining portions of FRQ are predicted to be unstructured. These potentially flexible random coils may eventually reach the catalytic site of a CK1a molecule bound to FRQ via the FCDs. A tightly bound, activated CK1a molecule corresponds to a high local kinase concentration, which may further facilitate phosphorylation in cis. It is not known whether the progressive phosphorylation of FRQ is entirely random or follows an obligate (or preferentially) sequential temporal pattern similar to the sequential phosphorylation of certain sites in PERIOD of Drosophila ${ }^{34}$.

FRH attenuates the overall phosphorylation kinetics of FRQ. As FRQ is phosphorylated at up to $\sim 100$ sites $^{4,5}$, the question arises how FRH can modulate the phosphorylation kinetics of FRQ at many different sites. Our data suggest that ATP hydrolysis by FRH may remodel the complex such that the access of CK1a to phosphorylation sites in FRQ is compromised. The ATPase cycle of FRH may thus control the temporal progression of the phosphorylation of FRQ that is crucial for the molecular timing process. Structural and functional analysis of recombinant clock components in vitro will help elucidating the detailed molecular mechanism.

Recently, it has been suggested that the ATPase of FRH is not required for the function of the circadian clock ${ }^{18}$. As FRH is essential for the viability of Neurospora, this conclusion was based on co-expression of two FRH alleles, one with a defect in the clock supplying the essential function of FRH, and the other with a defective ATPase. To be conclusive, it is mandatory that FRH does not form a homodimer in its interaction with FRQ dimers, as dimerization of FRH could lead to the formation of a FFC bearing one helicase null FRH and one clock null FRH, thereby allowing for cross-complementation ${ }^{18}$. We show that a monomeric mutant version of FRQ interacts with one molecule of FRH. As WT FRQ is a dimer or a higher oligomer ${ }^{19}$, the complex should contain stoichiometric amounts of FRH unless a specific mechanism would exclude binding of a second FRH molecule. The apparent molecular mass of the WT FFC and the reconstituted FFC $(\sim 670 \mathrm{kDa})$ suggests an assembly of at least two FRQ and two FRH molecules.

Recent mass spectrometry analysis suggested that the interaction of FRQ with CK1a decreases in the course of a day by an unknown mechanism ${ }^{5}$. We show here that hyperphosphorylation of FRQ compromises its interaction with CK1a. The FCD1/2 interaction, which is considerably weak, appears to be additionally stabilized by ionic interactions of the positively charged $\mathrm{N}$ terminus with the negatively charged middle and C-terminal portions of FRQ ${ }^{23}$. Hence, in the course of a circadian period, CK1a-dependent hyperphosphorylation of the positively charged $\mathrm{N}$ terminus may progressively weaken the FCD1/2 coiled-coil shifting of the CK1a-FRQ-binding equilibrium gradually towards dissociation.

In the core feedback loop of the Neurospora clock, the FFC complex transiently recruits and inactivates the circadian transcription factor WCC by facilitating its phosphorylation by bound $\mathrm{CK}_{1} \mathrm{a}^{29}$. The release of CK1a from hyperphosphorylated FRQ will functionally inactivate the FFC before proteolytic degradation of FRQ. Thus, inactivation of FRQ might be functionally more important for the circadian feedback loop than protein degradation. Degradation of FRQ might be required to maintain homeostasis, as accumulation of high levels of inactive FRQ could feedback (for example, via dephosphorylation) on the steady-state concentrations of active FRQ. It is not known whether clock proteins in other eukaryotic systems are regulated in similar manner. In the circadian clock of cyanobacteria KaiA and B proteins regulate independently of protein turnover autophosphorylation/dephosphorylation cycles and assembly state of KaiC hexamers ${ }^{35}$. KaiC oscillator contains two ATP-binding domains, CI and CII. The CII domain has autokinase/phosphatase activity crucial for the circadian timing process $^{36,37}$, and the slow ATPase of CI may act as an inputindependent timer $^{38}$. Regulation of FRQ by the two ATPases, CK1a and FRH, could be functionally similar to the regulation of KaiC by its CI and CII domains.

In summary, FRQ, the FRH ATPase and the CK1a kinase form a molecular timing device driven by two ATP-dependent processes. Hyperphosphorylation of FRQ is dependent on activated CK1a bound to FRQ. The ATPase cycle of FRH attenuates the phosphorylation kinetics on a circadian timescale leading to a progressive inactivation of the circadian inhibitor complex via dissociation of CK1a.

It is tempting to speculate that DEAD box-containing RNA helicases such as FRH could be more general ATPase-driven remodelling machines/modules that may, depending on their recruitment to specific complexes, drive conformational changes in double-stranded RNA structures and protein complexes in mechanistically similar manner.

\section{Methods}

Neurospora strains and culture conditions. Neurospora strains that were used in this study (WT, frq10, frq9, FRQ $\Delta \mathrm{NLS}^{20}, \Delta \mathrm{FCD} 1, \Delta \mathrm{FCD} 2$ (ref. 23), frq ${ }^{+}{ }_{\text {FLAG }} \mathrm{FRH}$

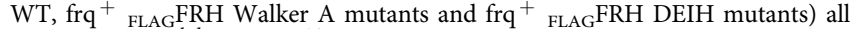
carried the ras- $1^{\text {bd }}$ mutation ${ }^{39}$. Conidial suspensions in $1 \mathrm{M}$ sorbitol were prepared from strains grown (5-7 days) on standard solid growth medium ( $2.2 \%$ agar, $0.3 \%$ glucose, $0.17 \%$ L-arginine, $1 \times$ Vogel's medium and $0.1 \%$ biotin). Standard growth medium for liquid cultures contained $2 \%$ glucose, $0.17 \% \mathrm{~L}$-arginine and $1 \times$

Vogel's medium. The cultures were grown in constant light at $25^{\circ} \mathrm{C}$ for $48 \mathrm{~h}$ if not indicated otherwise. Cycloheximide was added at a concentration of $10 \mu \mathrm{g}$ per $\mathrm{ml}$ and thiolutin was added to a final concentration of $12 \mu \mathrm{g}$ per ml where 
indicated. The race tube medium contained $2 \%$ agar, $0.1 \%$ glucose, $0.17 \%$ L-arginine, $1 \times$ Vogel's medium and $0.1 \%$ biotin. The race tubes were evaluated by densitometry and the Chrono $1 \mathrm{l}$ software (T. Roenneberg, Ludwig-MaximiliansUniversität Munich).

S. cerevisiae culture conditions. Yeast cells of the strain DS1-2b (Y2197; MAT, his3-4200 leu2- 41 trp1- 463 ura3-52, kindly provided by the Hurt group/University of Heidelberg Biochemistry Centre) for transformations with the respective vectors were grown in rich YPD medium. Transformation was performed as described previously ${ }^{40}$. Yeast strains were grown in selection medium: synthetic dextrose complete medium or synthetic raffinose complete medium. Synthetic raffinose complete medium was used if a construct containing a galactose promoter was present in a strain. Galactose was added to a final concentration of $1 \%$. Precultures were grown overnight at $30^{\circ} \mathrm{C}$ and 120 r.p.m. and used to inoculate new cultures that were grown to $\mathrm{OD}_{600}=0.5$ to further proceed with experimental procedures.

Plasmid construction. The Neurospora vector pMF309 (ref. 41) was used as a backbone for $c c g$-1-flag-frh constructs. A genomic PCR product was inserted as SpeI-EcoRI fragment (primers:F: $5^{\prime}$-actagtatggactacaaagacgatgatgacaaagattacaaggat gacgacgataaggggcgcgccatggacgacctctttgaggttttc- $3^{\prime}$ and R:5' $5^{\prime}$ tttctttcaacagtctgtatctgtaa gaattc- $3^{\prime}$ ). The Walker A and DEIH motif were changed by site-directed mutagenesis. Plasmids for expression of FRQ and FRH in yeast (generous gift of Hurt group/University of Heidelberg Biochemistry Centre) were based on: 4383-YCp111-P.ADH1-HA-RSA4 and 4045Y-Cp22-P.Gal-GFP-RSA4. PCR product amplified from complementary DNA was inserted into these vectors as NdeI-NotI and AscI-NotI fragments, respectively. CKla expressing yeast vectors were generated as described previously ${ }^{23}$.

Protein analysis. Extraction of Neurospora and S. cerevisiae native protein: Tissue/ cells were ground in the presence of liquid nitrogen using a pre-cooled pestle and mortar. The powder was suspended in extraction buffer $(50 \mathrm{mM} \mathrm{HEPES} / \mathrm{KOH}$ ( $\mathrm{pH}$ 7.4), $137 \mathrm{mM} \mathrm{NaCl}, 10 \%$ (v/v) glycerol, $5 \mathrm{mM}$ EDTA containing $1 \mathrm{mM}$ phenylmethylsulfonylfluoride (PMSF), $5 \mu \mathrm{g} \mathrm{ml}^{-1}$ leupeptin and $5 \mu \mathrm{g} \mathrm{ml}^{-1}$ pepstatin A ${ }^{29}$. Protein concentrations were determined by NanoDrop (PeqLab). Fourhundred micrograms of $N$. crassa and $100 \mu \mathrm{g}$ of S. cerevisiae extract were analysed by SDS-PAGE and transferred to a nitrocellulose membrane (GE Healthcare; $0.45 \mu \mathrm{m}, 10 \times 150 \mathrm{~cm})$ in $150 \mathrm{mM}$ glycine, $20 \mathrm{mM}$ Tris, $20 \%(\mathrm{v} / \mathrm{v})$ methanol and $0.08 \%(\mathrm{w} / \mathrm{v})$ SDS at $200 \mathrm{~mA}$ for $2.5 \mathrm{~h}$. The membrane was blocked with TBS and $5 \%$ milk (pH 7.4) before incubation with antibodies ${ }^{6,29}$. Antibodies against FRQ were used 1:20 diluted in 5\% milk and CK1a antibodies at dilution of 1:500 in 5\% milk ${ }^{6,23}$. An FRH (1:350 in 5\% milk) antibody was raised in rabbits against a peptide corresponding to amino-acid residues 2-18 (Pineda, Berlin). The MTR4 (1:20,000 in 5\% milk) antibody was a generous gift of David Tollervey, Edinburgh. Enhanced chemiluminescence signals were detected with X-ray films (Fuji). Several series of exposures were generated and quantified by using the ImageJ software (Rasband, W.S., ImageJ, U. S. National Institutes of Health). Uncropped scans of the most important western blots are shown in Supplementary Fig. 6.

Preparation of yeast denaturing extracts: a volume corresponding to $1.0 \mathrm{OD}_{600}$ was removed from exponentially growing cells. Cells were pelleted ( $3 \mathrm{~min}$ at 3,600 r.p.m.) and washed in $1 \mathrm{ml}$ water. Cells were resuspended in $150 \mu \mathrm{l}$ denaturing extraction buffer (1.85 M NaOH, $7.5 \% \beta$-mercaptoethanol; freshly prepared) and placed on ice for $10 \mathrm{~min}$. TCA $(55 \% ; 150 \mu \mathrm{l})$ was added and the mixture was incubated for $10 \mathrm{~min}$ on ice. Proteins were precipitated for $10 \mathrm{~min}$ at $14,000 \mathrm{~g}$ at $4{ }^{\circ} \mathrm{C}$. The pellet was resuspended in $40 \mu \mathrm{l} 0.1 \mathrm{M} \mathrm{NaOH}$ and $14 \mu \mathrm{l} 4 \times$ Laemmli sample buffer and incubated at $95^{\circ} \mathrm{C}$ for $5 \mathrm{~min}$. Aliquots corresponding to 0.5 $\mathrm{OD}_{600}$ of cells were analysed by SDS-PAGE ${ }^{42}$. All experiments and western blots were performed at least three times and one representative example is shown.

Purification of recombinant proteins. Recombinant His-tagged CK1a was pur ified from E. coli: A pre-culture was grown in LB medium containing ampicillin (100 ng $\mathrm{Il}^{-1}$ ) at $37^{\circ} \mathrm{C}$ overnight. The pre-culture was then diluted 1:100 and grown at $37^{\circ} \mathrm{C}$ to $0.3 \mathrm{OD}_{600}$, then temperature was lowered to $18^{\circ} \mathrm{C}$. The culture was induced with $0.1 \mathrm{mM}$ IPTG overnight and harvested at 4,000 r.p.m. for $20 \mathrm{~min}$. Following steps were performed at $4{ }^{\circ} \mathrm{C}$. $\mathrm{His}_{6}$-tagged kinase was isolated using standard Qiagen protocol for pQE30 vectors (QIA expressionist) with the following changes: the isolation of the $\mathrm{His}_{6}$-tagged kinase was done in a cold room using gravity or a pump with max $0.5 \mathrm{ml} \mathrm{min}^{-1}$ flow rate. Elution was performed in three steps with $1 \mathrm{ml}$ elution buffer and $1 \mathrm{~min}$ incubation between each step. The samples were immediately re-buffered into Neurospora protein extraction buffer (without glycerol) using PD-10 columns (GE Healthcare). Glycerol was added after re-buffering ${ }^{26}$ ). FRH (residues 1-1,106) and FRH $\Delta \mathrm{N}$ (residues 116-1,106) were expressed with an N-terminal $\mathrm{His}_{6}$-GST-tag followed by a TEV cleavage site in E. coli BL21(DE3) cells. Proteins were purified using a Ni-nitrilotriacetate (NTA) affinity step followed by TEV cleavage and heparin Sepharose chromatography (GE Healthcare). Size-exclusion chromatography was performed (Superdex 200, GE Healthcare) in $20 \mathrm{mM}$ HEPES $\mathrm{pH} 7.5,150 \mathrm{mM} \mathrm{NaCl}$ and $2 \mathrm{mM}$ DTT for final purification. FRQ (residues 1-989) was expressed with a N-terminal $\mathrm{His}_{6}-\mathrm{Z}$-tag ${ }^{43}$ followed by a TEV cleavage site in E. coli BL21(DE3) cells. FRQ was purified using a NTA affinity step followed by ion exchange. After ion exchange, the tag was removed by TEV cleavage. The uncleaved fusion protein and the TEV protease were removed by a second NTA affinity step. Size-exclusion chromatography was performed (Superdex 200, GE Healthcare) in $20 \mathrm{mM}$ HEPES, pH 7.5, $250 \mathrm{mM}$ $\mathrm{NaCl}, 10 \%$ glycerol and $2 \mathrm{mM}$ DTT for final purification ${ }^{13}$

Gel filtration. Neurospora extracts $(10-15 \mathrm{mg})$ or recombinant clock proteins $(5 \mathrm{mg})$ were loaded $(<0.25 \mathrm{ml})$ on a Superose 6 gel filtration column. Chromatography was performed at $4{ }^{\circ} \mathrm{C}$ at a flow rate of $0.5 \mathrm{ml} \mathrm{min}^{-1}$ and fractions of $0.5 \mathrm{ml}$ were collected.

In vitro phosphorylation assay. The phosphorylation reaction contained $50 \mathrm{mM}$ HEPES/KOH, pH 7.4, $150 \mathrm{mM} \mathrm{NaCl}, 10 \mathrm{mM} \mathrm{MgCl}_{2}, 10 \mathrm{mM}$ ATP, leupeptin $(2 \mu \mathrm{g}$ per $\mathrm{ml})$, pepstatin A $(2 \mu \mathrm{g}$ per $\mathrm{ml})$, PMSF $(1 \mathrm{mM})$, and PhosStop phosphatase inhibitor cocktail (Roche). Protein extract $(400 \mu \mathrm{g})$ was incubated with or without recombinant clock proteins (100 ng FRQ, $5 \mu \mathrm{g}{ }_{6 \mathrm{HIS}} \mathrm{CK} 1 \mathrm{a}$, if not indicated otherwise) in a final volume of $60 \mu \mathrm{l}$ at 4 or $30^{\circ} \mathrm{C}$. Laemmli sample $(4 \times ; 18 \mu \mathrm{l})$ buffer was added, followed by a 5 -min incubation at $95^{\circ} \mathrm{C}$. Aliquots were analysed by SDS gel electrophoresis followed by western Blot analysis with FRQ antibody.

Co-immunoprecipitation. CK1a IPs were performed as described previously ${ }^{23}$ Affinity-purified FRH antibody was bound to $30 \mu$ protein A Sepharose CL-4B beads (GE Healthcare) for $2 \mathrm{~h}$ at room temperature. The beads were washed twice with PBS containing protease and phosphatase (PhosStop, Roche) inhibitors. Cell lysate $(5 \mathrm{mg})$ prepared in PEX buffer was added to the beads. The reaction was filled up with PBS to $500 \mu \mathrm{l}$. The mixture was incubated overnight at $4{ }^{\circ} \mathrm{C}$ if not indicated otherwise. The beads were washed two times with PBS and the bound protein was eluted in $2 \times$ Laemmli sample buffer for $5 \mathrm{~min}$ at $95^{\circ} \mathrm{C}$. For IP of proteins with a FLAG-tag, $30 \mu \mathrm{l}$ M2 FLAG Sepharose (Sigma) was used.

GST pull-down experiment. GST-FRH (110 pmol) was incubated with 55, 110 or $220 \mathrm{pmol}$ of FRQ in $500 \mu \mathrm{l}$ binding buffer $(20 \mathrm{mM}$ HEPES/NaOH, $150 \mathrm{mM} \mathrm{NaCl}$, $5 \%$ glycerol and $2 \mathrm{mM}$ DTT, $\mathrm{pH} 7.5$ ) for $2 \mathrm{~h}$ on ice. After addition of glutathione beads, the samples were incubated for $2 \mathrm{~h}$ at $4{ }^{\circ} \mathrm{C}$, washed three times with $500 \mu \mathrm{l}$ binding buffer and eluted with $20 \mathrm{mM}$ HEPES/NaOH, $150 \mathrm{mM} \mathrm{NaCl}, 5 \%$ glycerol and $25 \mathrm{mM} \mathrm{GSH}, \mathrm{pH}$ 7.5. The eluted proteins were analysed by SDS-PAGE.

Quantitative real-time PCR. Total mRNA from ground frozen mycelia was prepared using peqGOLD TriFAST (PeqLab, Germany) and reverse transcription was performed using the QuantiTect Reverse Transcription Kit (Qiagen, Germany) following the manufacturer's instructions. Transcript levels were analysed by quantitative real-time PCR (RT-PCR) as described previously ${ }^{23}$. Sequences of primers and probes used for RT-PCR were: $f r q$ : F: $5^{\prime}$-ttgtaatgaaaggtgtccgaaggt- $3^{\prime}$; R: $5^{\prime}$-ggaggaagaagcggaaaacg- $3^{\prime}$; Probe: 6-FAM $5^{\prime}$-acctcccaatctccgaactcgcctg- $3^{\prime}$ (TAMRA); 28s rRNA: F: $5^{\prime}$-cctgttcgagcgtcatttca-3'; R: $5^{\prime}$-agcccgccactgattttg- $3^{\prime}$; 6-FAM $5^{\prime}$-ccatcaagctctgcttgcgttggg- $3^{\prime}$ (TAMRA).

\section{References}

1. Weber, F., Zorn, D., Rademacher, C. \& Hung, H. C. Post-translational timing mechanisms of the Drosophila circadian clock. FEBS Lett. 585, 1443 (2011).

2. Reischl, S. \& Kramer, A. Kinases and phosphatases in the mammalian circadian clock. FEBS Lett. 585, 1393 (2011).

3. Diernfellner, A. C. \& Schafmeier, T. Phosphorylations: making the Neurospora crassa circadian clock tick. FEBS Lett. 585, 1461 (2011).

4. Tang, C. T. et al. Setting the pace of the Neurospora circadian clock by multiple independent FRQ phosphorylation events. Proc. Natl Acad. Sci. USA 106, 10722 (2009)

5. Baker, C. L., Kettenbach, A. N., Loros, J. J., Gerber, S. A. \& Dunlap, J. C. Quantitative proteomics reveals a dynamic interactome and phase-specific phosphorylation in the Neurospora circadian clock. Mol. Cell 34, 354 (2009).

6. Gorl, M. et al. A PEST-like element in FREQUENCY determines the length of the circadian period in Neurospora crassa. EMBO J. 20, 7074 (2001).

7. Cheng, P., He, Q., He, Q., Wang, L. \& Liu, Y. Regulation of the Neurospora circadian clock by an RNA helicase. Genes Dev. 19, 234 (2005).

8. Putnam, A. A. \& Jankowsky, E. DEAD-box helicases as integrators of RNA, nucleotide and protein binding. Biochim. Biophys. Acta 1829, 884 (2013).

9. Linder, P. \& Fuller-Pace, F. V. Looking back on the birth of DEAD-box RNA helicases. Biochim. Biophys. Acta 1829, 750 (2013).

10. Jia, H. et al. The RNA helicase Mtr4p modulates polyadenylation in the TRAMP complex. Cell 145, 890 (2011).

11. Holub, P. et al. Air2p is critical for the assembly and RNA-binding of the TRAMP complex and the KOW domain of Mtr4p is crucial for exosome activation. Nucleic Acids Res. 40, 5679 (2012).

12. Wang, X., Jia, H., Jankowsky, E. \& Anderson, J. T. Degradation of hypomodified tRNA(iMet) in vivo involves RNA-dependent ATPase activity of the DExH helicase Mtr4p. RNA 14, 107 (2008) 
13. Weir, J. R., Bonneau, F., Hentschel, J. \& Conti, E. Structural analysis reveals the characteristic features of Mtr4, a DExH helicase involved in nuclear RNA processing and surveillance. Proc. Natl Acad. Sci. USA 107, 12139 (2010).

14. Guo, J., Cheng, P., Yuan, H. \& Liu, Y. The exosome regulates circadian gene expression in a posttranscriptional negative feedback loop. Cell 138, 1236 (2009).

15. Guo, J., Cheng, P. \& Liu, Y. Functional significance of FRH in regulating the phosphorylation and stability of Neurospora circadian clock protein FRQ. J. Biol. Chem. 285, 11508 (2010).

16. Cha, J., Yuan, H. \& Liu, Y. Regulation of the activity and cellular localization of the circadian clock protein FRQ. J. Biol. Chem. 286, 11469 (2011).

17. Shi, M., Collett, M., Loros, J. J., Dunlap, J. C. \& FRQ-interacting, RNA helicase mediates negative and positive feedback in the Neurospora circadian clock. Genetics 184, 351 (2010).

18. Hurley, J. M., Larrondo, L. F., Loros, J. J. \& Dunlap, J. C. Conserved RNA helicase FRH acts nonenzymatically to support the intrinsically disordered Neurospora clock protein FRQ. Mol. Cell 52, 832 (2013).

19. Cheng, P., Yang, Y., Heintzen, C. \& Liu, Y. Coiled-coil domain-mediated FRQFRQ interaction is essential for its circadian clock function in Neurospora. EMBO J. 20, 101 (2001).

20. Luo, C., Loros, J. J. \& Dunlap, J. C. Nuclear localization is required for function of the essential clock protein FRQ. EMBO J. 17, 1228 (1998).

21. Pause, A. \& Sonenberg, N. Mutational analysis of a DEAD box RNA helicase: the mammalian translation initiation factor eIF-4A. EMBO J. 11, 2643 (1992).

22. Hodge, C. A. et al. The Dbp5 cycle at the nuclear pore complex during mRNA export I: dbp5 mutants with defects in RNA binding and ATP hydrolysis define key steps for Nup159 and Gle1. Genes Dev. 25, 1052 (2011).

23. Querfurth, C. et al. Circadian conformational change of the Neurospora clock protein FREQUENCY triggered by clustered hyperphosphorylation of a basic domain. Mol. Cell 43, 713 (2011).

24. Peters, J. M., McKay, R. M., McKay, J. P. \& Graff, J. M. Casein kinase I transduces Wnt signals. Nature 401, 345 (1999)

25. Lee, H., Chen, R., Lee, Y., Yoo, S. \& Lee, C. Essential roles of CKIdelta and CKIepsilon in the mammalian circadian clock. Proc. Natl Acad. Sci. USA 106, 21359 (2009).

26. Tataroglu, O. et al. Glycogen synthase kinase is a regulator of the circadian clock of Neurospora crassa. J. Biol. Chem. 287, 36936 (2012).

27. Querfurth, C. et al. Posttranslational regulation of Neurospora circadian clock by CK1a-dependent phosphorylation. Cold Spring Harb. Symp. Quant. Biol. 72, 177 (2007).

28. Flotow, H. \& Roach, P. J. Role of acidic residues as substrate determinants for casein kinase I. J. Biol. Chem. 266, 3724 (1991).

29. Schafmeier, T. et al. Transcriptional feedback of Neurospora circadian clock gene by phosphorylation-dependent inactivation of its transcription factor. Cell 122, 235 (2005)

30. Yang, Y., Cheng, P., He, Q., Wang, L. \& Liu, Y. Phosphorylation of FREQUENCY protein by casein kinase II is necessary for the function of the Neurospora circadian clock. Mol. Cell Biol. 23, 6221 (2003).

31. Yang, Y., Cheng, P., Zhi, G. \& Liu, Y. Identification of a calcium/calmodulindependent protein kinase that phosphorylates the Neurospora circadian clock protein FREQUENCY. J. Biol. Chem. 276, 41064 (2001).

32. Huang, G. et al. Protein kinase A and casein kinases mediate sequential phosphorylation events in the circadian negative feedback loop. Genes Dev. 21, 3283 (2007)
33. Cruciat, C. M. et al. RNA helicase DDX3 is a regulatory subunit of casein kinase 1 in Wnt-beta-catenin signaling. Science 339, 1436 (2013).

34. Chiu, J. C., Ko, H. W. \& Edery, I. NEMO/NLK phosphorylates PERIOD to initiate a time-delay phosphorylation circuit that sets circadian clock speed. Cell 145, 357 (2011).

35. Nakajima, M. et al. Reconstitution of circadian oscillation of cyanobacterial KaiC phosphorylation in vitro. Science 308, 414 (2005).

36. Johnson, C. H., Stewart, P. L. \& Egli, M. The cyanobacterial circadian system: from biophysics to bioevolution. Annu. Rev. Biophys. 40, 143 (2011).

37. Kondo, T. A cyanobacterial circadian clock based on the Kai oscillator Cold Spring Harb. Symp. Quant. Biol. 72, 47 (2007).

38. Phong, C., Markson, J. S., Wilhoite, C. M. \& Rust, M. J. Robust and tunable circadian rhythms from differentially sensitive catalytic domains. Proc. Natl Acad. Sci. USA 110, 1124 (2013).

39. Belden, W. J. et al. The band mutation in Neurospora crassa is a dominant allele of ras-1 implicating RAS signaling in circadian output. Genes Dev. 21, 1494 (2007).

40. Malzahn, E., Ciprianidis, S., Kaldi, K., Schafmeier, T. \& Brunner, M. Photoadaptation in Neurospora by competitive interaction of activating and inhibitory LOV domains. Cell 142, 762 (2010).

41. Freitag, M., Hickey, P. C., Raju, N. B., Selker, E. U. \& Read, N. D. GFP as a tool to analyze the organization, dynamics and function of nuclei and microtubules in Neurospora crassa. Fungal Genet. Biol. 41, 897 (2004).

42. Knop, M. et al. Epitope tagging of yeast genes using a PCR-based strategy: more tags and improved practical routines. Yeast 15, 963 (1999).

43. Samuelsson, E., Moks, T., Nilsson, B. \& Uhlen, M. Enhanced in vitro refolding of insulin-like growth factor I using a solubilizing fusion partner. Biochemistry 33, 4207 (1994).

\section{Acknowledgements}

We thank Julia Kaim for excellent technical assistance. This work was supported by grants from the Deutsche Forschungsgemeinschaft: SFB 638 to M.B., DFG DI 1874/1-1 to A.D. M.B. is an investigator of CellNetworks.

\section{Author contributions}

M.B., A.D. and L.L. designed the research. L.L. performed the experiments. S.F. performed the purification of recombinant FRQ and FRH and the GST pull-down experiment. A.D. performed the cloning of the yeast vectors containing CKla and the purification of recombinant CK1a. M.B., A.D. and L.L. analysed the data. M.B., A.D. and L.L. wrote the paper.

\section{Additional information}

Supplementary Information accompanies this paper at http://www.nature.com/ nature communications

Competing financial interests: The authors declare no competing financial interests.

Reprints and permission information is available online at http://npg.nature.com/ reprintsandpermissions/

How to cite this article: Lauinger, L. et al. The RNA helicase FRH is an ATP-dependent regulator of CK1a in the circadian clock of Neurospora crassa. Nat. Commun. 5:3598 doi: $10.1038 /$ ncomms4598 (2014) 